\title{
Ventricular Sigmoid Septum as a Risk Factor for Anthracycline-Induced Cancer Therapeutics-Related Cardiac Dysfunction in Patients with Malignant Lymphoma
}

\author{
Takafumi Nakayama ${ }^{1}$, Yoshiko Oshima ${ }^{2}$, Yasuhiro Shintani ${ }^{1}$, Junki Yamamoto ${ }^{1}$, Masashi \\ Yokoi $^{1}$, Tsuyoshi Ito ${ }^{1}$, Kazuaki Wakami ${ }^{1}$, Shuichi Kitada ${ }^{1}$, Toshihiko Goto ${ }^{1}$, Shigeru \\ Kusumoto $^{1}$, Tomonori Sugiura ${ }^{1}$, Shinsuke Iida ${ }^{1}$, and Yoshihiro Seo ${ }^{1}$ \\ ${ }^{1}$ Nagoya City University \\ ${ }^{2}$ Toyokawa City Hospital
}

September 25, 2021

\begin{abstract}
ABSTRACT Background Identifying risk factors for cancer therapeutics-related cardiac dysfunction (CTRCD) is essential for early detection and prompt initiation of medial therapy for CTRCD. There has been no study investigating whether the sigmoid septum is a risk factor for anthracycline-induced CTRCD. Methods We enrolled 167 patients with malignant lymphoma who received a CHOP-like regimen from January 2008 to December 2017 and underwent both baseline and followup echocardiography. Patients with LVEF [?] 50\% were excluded. CTRCD was defined as [?] 10\% decline in LVEF and LVEF $<50 \%$ after chemotherapy. The angle between the anterior-wall of the aorta and the ventricular septal surface (ASA) was measured to quantify the sigmoid septum. Results CTRCD was observed in 36 patients (22\%). The average LVEF and GLS were lower (61.6 vs. $65.0 \%, 18.7$ vs. 20.3), LV mass index was higher (101.1 vs. $92.8 \mathrm{~g} / \mathrm{m} 2)$, and ASA was smaller (112.1 vs. 119.4 degree) in patients with CTRCD. In a multivariable Cox proportional hazard analysis, GLS (HR per 1\% decrease 1.20 , 95\% CI 1.07-1.35, P = 0.002), ASA (HR per 1 degree increase $0.97,95 \%$ CI 0.95-0.99, P = 0.003) and the history of ischemic heart disease (HR 5.13, 95\% CI 1.94-13.56, P = 0.001) were identified as the independent determinants of CTRCD. C-statistics analysis and integrated discrimination improvement proved the significant incremental value of ASA for developing CTRCD. Conclusion Smaller ASA was the independent risk factor and had significant incremental value for CTRCD in patients with malignant lymphoma who received the CHOP-like regimen.
\end{abstract}

\section{Original Investigations}

\section{Title:}

Ventricular Sigmoid Septum as a Risk Factor for Anthracycline-Induced Cancer Therapeutics-Related Cardiac Dysfunction in Patients with Malignant Lymphoma

\section{Authors:}

Takafumi Nakayama $^{\mathrm{a}}, \mathrm{MD}, \mathrm{PhD}$, Yoshiko Oshima ${ }^{\mathrm{b}}, \mathrm{MD}$, Yasuhiro Shintani ${ }^{\mathrm{a}}, \mathrm{MD}, \mathrm{PhD}$, Junki Yamamoto $^{\mathrm{a}}$, MD, PhD, Masashi Yokoi ${ }^{a}$, MD, Tsuyoshi Ito ${ }^{\mathrm{a}}, \mathrm{MD}, \mathrm{PhD}$, Kazuaki Wakami ${ }^{\mathrm{a}}$, MD, PhD, Shuichi Kitada ${ }^{\mathrm{a}}$, $\mathrm{MD}, \mathrm{PhD}$, Toshihiko Goto ${ }^{\mathrm{a}}$ MD, PhD, Shigeru Kusumoto ${ }^{\mathrm{b}}, \mathrm{MD}, \mathrm{PhD}$, Tomonori Sugiura ${ }^{\mathrm{a}}, \mathrm{MD}, \mathrm{PhD}$, Shinsuke Iida ${ }^{\mathrm{b}}, \mathrm{MD}, \mathrm{PhD}$, Yoshihiro $\mathrm{Seo}^{\mathrm{a}}, \mathrm{MD}, \mathrm{PhD}$

\section{Affiliations:}

a Department of Cardiology, Nagoya City University Graduate School of Medical Sciences 
1-Kawasumi, Mizuho-cho, Mizuho-ku, Nagoya, 467-8601, Japan

b Department of Hematology and Oncology, Nagoya City University Graduate School of Medical Sciences

1-Kawasumi, Mizuho-cho, Mizuho-ku, Nagoya, 467-8601, Japan

Corresponding author contact information:

Correspondence: Yoshihiro Seo, MD, PhD

Department of Cardiology, Nagoya City University Graduate School of Medical Sciences

1-Kawasumi, Mizuho-cho, Mizuho-ku, Nagoya, 467-8601, Japan

Phone: +81-52-853-8221; Fax: +81-52-852-3796

E-mail: yo-seo@med.nagoya-cu.ac.jp

\section{Running head:}

Sigmoid-septum and CTRCD in ML patients

Total word count:

4,439 words from introduction to references.

\section{Declarations of interest:}

This research did not receive any specific grant from funding agencies in the public, commercial, or not-forprofit sectors.

\section{ABSTRACT \\ Background}

Identifying risk factors for cancer therapeutics-related cardiac dysfunction (CTRCD) is essential for early detection and prompt initiation of medial therapy for CTRCD. There has been no study investigating whether the sigmoid septum is a risk factor for anthracycline-induced CTRCD.

\section{Methods}

We enrolled 167 patients with malignant lymphoma who received a CHOP-like regimen from January 2008 to December 2017 and underwent both baseline and follow-up echocardiography. Patients with LVEF [?] $50 \%$ were excluded. CTRCD was defined as [?] $10 \%$ decline in LVEF and LVEF < 50\% after chemotherapy. The angle between the anterior-wall of the aorta and the ventricular septal surface (ASA) was measured to quantify the sigmoid septum.

\section{Results}

CTRCD was observed in 36 patients (22\%). The average LVEF and GLS were lower (61.6 vs. 65.0\%, 18.7 vs. 20.3), LV mass index was higher (101.1 vs. $92.8 \mathrm{~g} / \mathrm{m}^{2}$ ), and ASA was smaller (112.1 vs. 119.4 degree) in patients with CTRCD. In a multivariable Cox proportional hazard analysis, GLS (HR per $1 \%$ decrease $1.20,95 \%$ CI 1.07-1.35, $\mathrm{P}=0.002$ ), ASA (HR per 1 degree increase $0.97,95 \%$ CI 0.95-0.99, $\mathrm{P}=$ 0.003 ) and the history of ischemic heart disease (HR 5.13, 95\% CI 1.94-13.56, $\mathrm{P}=0.001$ ) were identified as the independent determinants of CTRCD. C-statistics analysis and integrated discrimination improvement proved the significant incremental value of ASA for developing CTRCD.

\section{Conclusion}

Smaller ASA was the independent risk factor and had significant incremental value for CTRCD in patients with malignant lymphoma who received the CHOP-like regimen.

\section{Keywords:}


Cancer therapeutics-related cardiac dysfunction, anthracycline-induced cardiotoxicity, sigmoid septum, aorto-septal angle

\author{
ABBREVIATIONS \\ ASA $=$ the angle between the anterior-wall of the aorta and the ventricular septal surface (aorto-septal \\ angle) \\ $\mathrm{B}-\mathrm{M}$ ratio $=$ the ratio of basal to mid interventricular septal diameter (basal-mid ratio) \\ $\mathrm{CHOP}=$ cyclophosphamide, doxorubicin, vincristine and prednisolone \\ $\mathrm{CI}=$ confidence interval \\ CTRCD $=$ Cancer therapeutic-related cardiac dysfunction \\ DUST $=$ discrete upper septal thickening \\ $\mathrm{HR}=$ hazard ratio \\ IDI = integrated discrimination improvement \\ $\mathrm{LVEF}=$ left ventricular ejection fraction \\ $\mathrm{LVMi}=$ left ventricular mass index \\ $\mathrm{ML}=$ malignant lymphoma \\ $\mathrm{NRI}=$ net reclassification index \\ $\mathrm{R}-\mathrm{CHOP}=$ rituximab, cyclophosphamide, doxorubicin, vincristine, and prednisolone \\ INTRODUCTION
}

Anthracycline is an effective chemotherapeutic agent for several kinds of malignant tumors, particularly in patients with malignant lymphoma (ML) and breast cancer. ${ }^{1-3}$ In contrast, anthracycline-induced cancer therapeutic-related cardiac dysfunction (CTRCD), defined by a decline in left ventricular ejection fraction (LVEF), often causes poor clinical outcomes and low quality of life in patients with cancer. ${ }^{4-9}$ Identification of baseline risk factors for CTRCD is vital for early detection and prompt initiation of medical therapy, which are essential for improving left ventricular (LV) contraction after CTRCD develops. ${ }^{10-12}$ Several baseline risk factors have been clarified in previous studies, including baseline LVEF, global longitudinal strain (GLS), age, general cardiovascular risk factors, a history of cardiovascular disease. ${ }^{4,11,13-15}$ Recently, LV hypertrophy was recognized as a risk factor for CTRCD in ML patients ${ }^{16}$, suggesting LV morphologic remodeling also represents an accumulated myocardial injury that may cause a vulnerable condition for CTRCD. On the other hand, the sigmoid septum, known as a synonym of discrete upper septal thickening, is considered one of LV morphologic remodeling. The previous investigation reported that the sigmoid septum was significantly related to LV diastolic dysfunction. ${ }^{17}$ However, no one clarified the association of the sigmoid septum with the incidence of CTRCD.

In the current study, we focused on the presence of the sigmoid septum and aimed to reveal its impact on anthracycline-induced CTRCD in patients with ML who received the CHOP-like regimen.

\title{
MATERIALS AND METHODS
}

\section{Study population}

All ML patients who received the CHOP-like regimen (cyclophosphamide + doxorubicin + vincristine + prednisolone \pm rituximab) from January 2008 to December 2017 at Nagoya City University Hospital were retrospectively reviewed. The dosing and schedule for the CHOP-like regimen were standardized in this cohort. From this group, patients who underwent a baseline echocardiographic examination and at least one or more follow-up examinations were enrolled in the current study (Figure 1). Patients with LVEF $<50 \%$ 
at baseline but no echocardiographic images of adequate quality were excluded. The current study was the sub-analysis of a previous study in which we described the clinical features of anthracycline-induced CTRCD 18 .

\section{Study protocol}

We retrospectively collected patients' clinical characteristics, including age, sex, body mass index, general cardiovascular risk factors, past history, laboratory data, chemotherapeutic regimen, performance status, and echocardiographic parameters from their medical records. The chemotherapeutic regimen, performance status, and dose of anthracycline were assessed by two hematologists. Patients with intravascular large B-cell lymphoma (IVL) and primary mediastinal large B cell lymphoma (PMLBCL) were enrolled as a subtype of diffuse large B-cell lymphoma (DLBCL) in the current study. Echocardiographic parameters and the incidences of symptomatic heart failure and cardiovascular death were obtained and assessed by two cardiologists in a blinded manner. In the current study, CTRCD was defined as a decline in LVEF of $10 \%$ or more from baseline and LVEF $<50 \%$ in the follow-up study. ${ }^{9}$

Cardiovascular risk factors were defined as hypertension, dyslipidemia, diabetes mellitus, and a history of smoking. Each cardiovascular risk factor was defined by the following criteria: hypertension, as a record of a history of hypertension or being on antihypertensive therapy; dyslipidemia, as a record of a history of dyslipidemia, serum low-density lipoprotein cholesterol level [?] $140 \mathrm{mg} / \mathrm{dL}$, or being on cholesterol-lowering treatment; and diabetes mellitus, as a record of a history of diabetes mellitus, HbA1c [?] 6.5\%, or being on blood glucose-lowering therapy. History of ischemic heart disease was defined as a history of myocardial infarction, angina pectoris, or coronary artery revascularization. The current study was approved by the ethics committee of our institution (No. 60-19-0125) and was carried out according to the principles of the Helsinki Declaration.

\section{Echocardiography}

Echocardiographic data were measured according to the recommendations of the American Society of Echocardiography ${ }^{19}$. However, avoiding the region of the sigmoid septum, the LV end-diastolic diameter (LVDd), interventricular septal wall thickness (IVSd), and posterior wall thickness (PWd) were measured at a mid-ventricular position. LVEF was calculated by the disk summation method using the TomTec imaging system (TomTec Imaging System GmbH, Munich, Germany). To avoid a misdiagnosis of CTRCD, we remeasured LVEF, which was between $40 \%$ to $60 \%$ by the first measurer, by the second measurer (the other of the first measurer) to assess the accuracy of diagnosis. LV wall thickness was defined as the mean IVSd and PWd. LV mass was calculated using the following formula (10): 1.04x $\left[(\mathrm{LVDd}+\mathrm{IVS}+\mathrm{PWd})^{3}-\mathrm{LVDd}^{3}\right] \mathrm{x} 0.8$ +0.6 . The grading of valvular diseases was assessed according to the guidelines of the Japanese Circulation Society. ${ }^{20}$

As morphological parameters, we calculated the ratio of basal to mid interventricular septal diameter (basalmid ratio, B-M ratio), and measured the angle between the anterior-wall of the aorta and the ventricular septal surface (aorto-septal angle, ASA) in the end diastolic phase to quantify discrete upper septal thickening (Figure 2).

\section{Statistical analysis}

Continuous variables were expressed as mean +- standard deviation. Continuous variables were compared using unpaired Student's t-test. Welch's test was used to compare continuous variables with heteroscedasticity. Categorical variables were analyzed using Pearson's chi-squared test. Cox proportional hazards analyses were performed to identify risk factors for the primary endpoint. The common logarithmic conversion was used when BNP was assessed in Cox proportional hazard analyses.

The primary endpoint was the incidence of CTRCD, and the initial time-point for each survival analysis was the first day of the CHOP-like regimen. Follow-up censoring of all participants was the day of the first detection of CTRCD or final echocardiographic examination. Clinical variables recognized as risk factors for CTRCD were analyzed using univariate Cox proportional hazards analyses. The GLS value was converted to 
an absolute value when assessed in Cox proportional hazard analyses, Kaplan-Meier analyses and Logistic regression analyses. In the first multivariable Cox proportional hazards analysis, echocardiographic parameters with $\mathrm{p}<0.05$ in the univariate analyses were analyzed to clarify the independent echocardiography-derived determinants of the event (multivariable analysis 1). Next, the independent echocardiographic parameters were adjusted by the clinical parameters with $\mathrm{p}<0.05$ in the univariate analyses as multivariable analysis 2. Multivariable Cox proportional hazard analyses were assessed by the forward stepwise method. Youden's index derived from receiver operating characteristics (ROC) curves were used to determine the cut-off value for risk factors for the primary endpoint. Event-free survival curves using the cut-off values were constructed by Kaplan-Meier analyses, and the log-rank test was used to assess the significance between curves.

To assess the incremental value of newly recognized risk factors for CTRCD, we defined two categorized models: model 1 included the risk factors that were already considered risk factors in a previous study and were recognized independently for CTRCD in the current study, whereas model 2 had all independent risk factors identified in the current study, including newly recognized risk factors. First, we compared the c-statistics of each model, which was calculated as an area under the curve (AUC) to assess the utility of new risk factors. Second, we used the net reclassification index (NRI) and integrated discrimination improvement (IDI) to evaluate the incremental value of risk factors newly identified in this study ${ }^{21}$. In these analyses, patients with a probability [?] 0.5 in logistic regression analyses in each model were considered to have CTRCD.

NRI was calculated according to the following formula:

$\mathrm{NRI}=[\mathrm{P}(\operatorname{up} \mid \mathrm{D}=1)-\mathrm{P}(\operatorname{down} \mid \mathrm{D}=1)]-[\mathrm{P}(\mathrm{up} \mid \mathrm{D}=0)-\mathrm{P}(\operatorname{down} \mid \mathrm{D}=0)]$

where $\mathrm{P}$ is the proportion of patients, "up" is upward movement defined as a change into a higher probability of CTRCD based on model 1, "down" is downward movement defined as a change in the opposite direction, and D is the classification of whether CTRCD develops (developing CTRCD $=1$, not developing CTRCD $=0)$.

IDI was calculated according to the following formula:

IDI $=\left[\left(P_{\text {extended }} \mid \mathrm{D}=1\right)-\left(\mathrm{P}_{\text {basic }} \mid \mathrm{D}=1\right)\right]-1\left[\left(\mathrm{P}_{\text {extended }} \mid \mathrm{D}=0\right)-\left(\mathrm{P}_{\text {basic }} \mid \mathrm{D}=0\right)\right]$

where $P_{\text {extended }} \mid D=1$ and $P_{\text {extended }} \mid D=0$ are the average values of the predicted probability of developing CTRCD in model 2 for the group that developed CTRCD and the group that did not, respectively; and $\mathrm{P}_{\text {basic }} \mid \mathrm{D}=1$ and $\mathrm{P}_{\text {basic }} \mid \mathrm{D}=0$ are the average values of the predicted probability of developing CTRCD in model 1 for the group that developed CTRCD and the group that did not, respectively.

Two-sided $\mathrm{P}<0.05$ was considered significant. All analyses were performed using SPSS ver. 26 (SPSS, Inc., Chicago, IL, USA).

\section{RESULTS}

\section{Study population}

Of 443 enrolled patients, 14 patients without baseline echocardiography data and 185 patients without followup echocardiography data were excluded. Fourteen patients with LVEF $<50 \%, 62$ patients whose GLS could not be quantified by tri-plane, and one patient without any echocardiographic images available for analysis were also excluded. In the comparison between the patients who underwent GLS measurements and those who were excluded because they could not have GLS measurements, no significant differences were observed in basic data and laboratory data (Table S1).

Finally, 167 patients were eligible for analysis in the current study (Figure 1). CTRCD was observed in 36 patients (22\%) during the follow-up period, which was a median 305 days (IQR: 171-837). The mean and median number of follow-up echocardiographic examinations were $3.0+-2.6$ and 2 (IQR, 1-4). Median interval duration of all echocardiographic follow-up study was 112 days (IQR, 50-238).

\section{Baseline characteristics}


The baseline characteristics of eligible patients are summarized in Table 1. No significant differences were found in baseline characteristics between patients with and without CTRCD except for the prevalence of prior ischemic heart disease. Most patients (83\%) received the R-CHOP regimen, and the remaining received the CHOP regimen. Patients with DLBCL were $67 \%(\mathrm{n}=111)$, including 4 and 2 patients with IVL and PMLBCL, respectively. The mean cumulative dose of doxorubicin was almost within the accurate range of 291.5 +- $95.3 \mathrm{mg} / \mathrm{m}^{2}$ (Table 1 ).

\section{Baseline echocardiographic parameters}

Echocardiographic parameters at baseline for the entire cohort are presented in Table 2. Mean LVEF was $64.3+-6.2 \%$ and 29 patients $(18 \%)$ had valvular disease [?] moderate ( $\mathrm{n}=5$ aortic regurgitation, $\mathrm{n}=6$ mitral regurgitation, $\mathrm{n}=23$ tricuspid regurgitation, $\mathrm{n}=1$ aortic stenosis). As a parameter of discrete upper septal thickening, the B-M ratio and ASA were 1.28 +- 0.24 and 117.8 +- 13.9 degree (Table 2).

We found significant differences between patients with and without CTRCD in LVEF $(61.6+-5.3 \%$ vs. $65.0+-6.2 \%, \mathrm{P}=0.003)$, GLS (18.7 +- $2.9 \%$ vs. $20.3+-3.4 \%, \mathrm{P}=0.008)$, LV mass index (LVMi) (101.1 $+-18.5 \mathrm{~g} / \mathrm{m}^{2}$ vs. $\left.92.8+-23.1 \mathrm{~g} / \mathrm{m}^{2}, \mathrm{P}=0.047\right)$, B-M ratio $(1.35+-0.22$ vs. $1.26+-0.24, \mathrm{P}=0.029)$, and ASA $(112.1+-14.2$ degree vs. $119.4+-13.4$ degree, $\mathrm{P}=0.005)$.

\section{Risk factors for CTRCD}

In univariate Cox proportional hazard analyses, LVEF, GLS, LVMi, and ASA were significantly associated with the primary endpoint in baseline echocardiographic parameters. In regard to clinical baseline characteristics, age, performance status [?] 2, and history of ischemic heart disease were associated with the primary endpoint (Table 3). In the multivariable Cox proportional hazard analysis 1, GLS (HR per 1\% decrease 1.19, $95 \%$ CI $1.06-1.33, \mathrm{P}=0.003$ ) and ASA (HR per 1 degree increase $0.97,95 \%$ CI $0.95-0.99, \mathrm{P}=0.004$ ) were identified as independent risk factors. The multivariable analysis 2 clarified GLS (HR per 1\% decrease 1.20, $95 \%$ CI 1.07-1.35, $\mathrm{P}=0.002$ ), ASA (HR per 1 degree increase $0.97,95 \%$ CI $0.95-0.99, \mathrm{P}=0.003$ ) and the history of ischemic heart disease (HR 5.13, 95\% CI 1.94-13.56, $\mathrm{P}=0.001$ ) as the independent risk factors for the primary endpoint (Table 3 ).

ROC curves for GLS and ASA regarding the incidence of CTRCD are presented in Figure S1. Cut-off values were $-19.4 \%$ for GLS, and 117 degree for ASA. The AUC, sensitivity, and specificity at each cut-off value were $0.65,0.61$, and 0.63 for GLS; 0.67, 0.72, and 0.61 for ASA, respectively (Figure S1). The Kaplan-Meier estimate curves for GLS and ASA are shown in Figure 3, with significant differences observed between each cut-off value.

\section{Incremental value of newly recognized risk factor}

According to the independent risk factors for CTRCD in this study, we defined model 1 as consistent with a history of ischemic heart disease and GLS, and model 2 as consistent with a history of ischemic heart disease, GLS, and ASA. ROC curves for probability in model 1 and model 2 are presented in Figure 4. The C-statistic (AUC) for model 2 was 0.72 , which is superior to the 0.67 of model 1 . The NRI and IDI were calculated between model 1 and model 2. The reclassification table for developing CTRCD is presented in Table 4 , with a probability cut-off of 0.5 . The NRI was (1 of $36-0$ of 36$)-(2$ of $131-0$ of 131$)=0.028$ $-0.015=0.013(95 \%$ CI $-0.004-0.029 ; \mathrm{P}=0.95)$, and the IDI was $(0.323-0.293)-(0.186-0.194)=0.030$ $+0.008=0.038(95 \%$ CI $0.009-0.068 ; \mathrm{P}=0.017)$.

\section{DISCUSSION}

The current study demonstrated that ASA was the independent risk factor for CTRCD in patients with ML who received the CHOP-like regimen, and ASA had a significant incremental value to predict the development of CTRCD. Any other clinical characteristics at baseline and dose of anthracycline were not independent risk factors for CTRCD. Furthermore, when excluding patients with valvular diseases $>$ moderate from the analyses, ASA was still an independent risk factor for developing CTRCD (Table S2). 
The relationship of almost all of echocardiographic parameters, except LVEF and GLS, and CTRCD has not been examined previously. To the best of our knowledge, this investigation is the first study to detect the impact of morphological parameters in echocardiography on CTRCD. In our study, history of ischemic heart disease and lower GLS were risk factors for CTRCD, consistent with previous studies ${ }^{11,15,22,23}$. We also found that smaller ASA was the new risk factor for CTRCD.

\section{Smaller ASA / ventricular sigmoid septum}

Several terms have been used to describe the sigmoid septum, including discrete upper septal thickening (DUST), subaortic ventricular septal bulge, and discrete upper septal knuckle. In general, the sigmoid septum is frequently observed in the elderly population ${ }^{24-26}$, although some investigators have reported this geometric change as part of the spectrum of hypertrophic cardiomyopathy, because of LV outlet obstruction 27-29. Therefore, the sigmoid septum is considered to be the result of aging-related changes associated with aortic atherosclerosis or thickening and calcification of the aortic or mitral valve. ${ }^{30,31}$ In the current study, higher age and higher body mass index were independently associated with the presence of sigmoid septum, as defined by the cut-off value of the ROC curve for CTRCD, in the logistic regression analysis (Table S3A). In the current study, in univariable Cox regression analysis, age was one of the significantly associated factors with CTRCD, consistent with previous reports. However, after multivariable analysis, ASA was proven as the independent risk factor for CTRCD, while age was not the independent determinant. That seemed to be why morphologic change as a result of aging was more specific and important for developing CTRCD than aging itself.

The clinical and prognostic significance of the sigmoid septum has not been clarified previously. A large prospective observational trial could not show the effects of the sigmoid septum on exercise capacity in a healthy population. ${ }^{32}$ In addition, a large retrospective study reported that the sigmoid septum was not independently associated with adverse clinical outcomes. ${ }^{33}$ However, the criteria for the sigmoid septum in these previous studies were based on the B-M ratio, which is rather a representative parameter of DUST. One of the reasons why the sigmoid septum had a clinically significant meaning in our study was the use of ASA for the quantification of the sigmoid septum. In the previous study, ASA was found to be the independent predictor of LV diastolic dysfunction quantified by LV peak diastolic longitudinal strain rate in healthy older subjects, otherwhile, age was not independently associated with LV diastolic dysfunction. ${ }^{17}$ We anticipate ASA could be an early predictor for LV dysfunction, resulting in being a risk factor for CTRCD.

\section{GLS and a history of ischemic heart disease}

Lower LVEF and smaller GLS in patients with normal LVEF were already recognized as risk factors for anthracycline-induced CTRCD. ${ }^{11,13,14,18}$ In the current study, LVEF was not an independent determinant for anthracycline-induced CTRCD in the multivariable analysis, probably because GLS can detect myocardial injury more sensitively than LVEF. ${ }^{34,35}$ History of ischemic heart disease is also considered a risk factor for CTRCD. ${ }^{23}$ Both decreased GLS and prior ischemic heart disease at baseline are generally considered to be associated with LV myocardial injury, even during LVEF is within normal limit. In the logistic regression analysis for lower GLS below the cut-off value in the current study, performance status [?] 2, higher total bilirubin and lower LVEF were the independent determinants (Table S3B). Considering a history of general risk factors of ischemic heart disease themselves including hypertension, hyperlipidemia, diabetes mellitus, and smoking were not identified as risk factors for CTRCD in this study, a history of ischemic heart disease might indicate the myocardial damage more directly.

\section{Possible theory for developing CTRCD}

Based on our main findings, echocardiography-proven lower LV longitudinal contraction and LV morphological changes were independent risk factors for CTRCD. These parameters are considered to progress according to cumulative exposure to myocardial tissue damage, which may be associated with several kinds of factors such as aging, obesity, performance status, and liver function (Table S3). Furthermore, although CTRCD defined by a decline in LVEF can recover to preserved LVEF by medical treatments for heart failure ${ }^{11}$, anthracycline-induced cardiomyocyte toxicity is pathologically considered irreversible and cumulative. ${ }^{36}$ 
Taken these mechanisms together, the frequency of anthracycline-induced CTRCD is defined upon the accumulated myocardial tissue damage that is the sum of the underlying myocardial damage before chemotherapy and additional myocardial tissue injury by anthracycline. We can guess the degree of myocardial tissue damage at baseline by lower GLS and the smaller ASA, as the most relevant risk factors for anthracycline-induced CTRCD.

\section{Study limitations}

The current study has some limitations. First, it was a single-center, retrospective study, and enrollment volume was limited. Second, a relatively large number of patients who did not undergo baseline or follow-up echocardiography were excluded. The differences between the group who underwent analyses and the group who excluded because of the absence of baseline or follow-up echocardiography are presented in Table S4. Baseline age was higher in the analyzed group, suggesting the attending physicians ordered echocardiographic examinations more frequently to potentially high-risk patients, possibly resulting in the high prevalence of CTRCD in the current study. Third, the timing of follow-up echocardiography also varied depending on the attending physician. The median interval duration between the follow-up examinations was 112days, suggesting relatively well-quality follow-ups were performed. However, there was a possibility that the diagnosis of CTRCD was delayed or not be made. And fourth, the sensitivity and specificity of cut-off values evaluated in the same cohort used for derivation are biased due to over-optimism. Further study is needed to assess the prognostic value of ASA in a prospective cohort.

\section{CONCLUSION}

In addition to lower GLS, decreased ASA was found to be a new independent risk factor for anthracyclineinduced CTRCD in patients with ML who received a CHOP-like regimen.

\section{DECLARATIONS OF INTEREST}

This research did not receive any specific grant from funding agencies in the public, commercial, or not-forprofit sectors.

\section{AUTHOR CONTRIBUTIONS}

Takafumi Nakayama and Yoshihiro Seo designed and performed the research. Takafumi Nakayama wrote the paper, and Yoshihiro Seo oversaw the paper and the whole research. Takafumi Nakayama, Yoshiko Oshima and Junki Yamamoto collected the clinical data. Yasuhiro Shintani, Masashi Yokoi, Tsuyoshi Ito, Kazuaki Wakami, Shuichi Kitada, Toshihiko Goto, Shigeru Kusumoto, Tomonori Sugiura, Shinsuke Iida and Yoshihiro Seo revised the paper critically. All authors approved the submission of the paper of final version.

\section{FIGURE LEGENDS}

Figure 1.

Study flow diagram of patients' enrollment.

Figure 2.

Representative echocardiographic images when morphological parameters associated with the sigmoid septum were measured. Basal-mid ratio was defined as the ratio of basal to mid interventricular septal diameter (A), aorto-septal angle was defined as the angle between the anterior-wall of the aorta and the ventricular septal surface (B).

Figure 3.

Kaplan-Meier curves for CTRCD. CTRCD-free survival was calculated according to each cut-off value for GLS (A) and ASA (B) determined by receiver operating characteristic curves. Event-free survival rates were significantly lower in groups with lower GLS and smaller ASA.

Figure 4 . 
C-statistics for developing CTRCD. (A) Model 1 includes a history of ischemic heart disease and GLS as already known risk factors. (B) Model 2 includes a history of ischemic heart disease, GLS, and incremental variables of ASA. The AUC of models 1 and 2 were 0.67 and 0.72 , respectively.

Figure S1.

Receiver operating characteristic curves to determine the cut-off values of GLS (A) and ASA (B) for KaplanMeier analyses. Areas under the curves (AUCs) were 0.65 for GLS and 0.67 for ASA. Sensitivities/specificities were $0.61 / 0.63$ and $0.72 / 0.61$ in GLS and ASA, respectively.

\section{REFERENCES}

1. Coiffier B, Lepage E, Briere J, et al. CHOP chemotherapy plus rituximab compared with CHOP alone in elderly patients with diffuse large-B-cell lymphoma. N Engl J Med. 2002;346(4):235-242.

2. Launchbury AP, Habboubi N. Epirubicin and doxorubicin: a comparison of their characteristics, therapeutic activity and toxicity. Cancer Treat Rev. 1993;19(3):197-228.

3. Fisher RI, Gaynor ER, Dahlberg S, et al. Comparison of a standard regimen (CHOP) with three intensive chemotherapy regimens for advanced non-Hodgkin's lymphoma. N Engl J Med. 1993;328(14):1002-1006.

4. Doroshow JH. Doxorubicin-induced cardiac toxicity. N Engl J Med. 1991;324(12):843-845.

5. Felker GM, Thompson RE, Hare JM, et al. Underlying causes and long-term survival in patients with initially unexplained cardiomyopathy. $N$ Engl J Med. 2000;342(15):1077-1084.

6. Henriksen PA. Anthracycline cardiotoxicity: an update on mechanisms, monitoring and prevention. Heart. 2018;104(12):971-977.

7. Armenian SH, Lacchetti C, Barac A, et al. Prevention and Monitoring of Cardiac Dysfunction in Survivors of Adult Cancers: American Society of Clinical Oncology Clinical Practice Guideline. J Clin Oncol.2017;35(8):893-911.

8. Campia U, Moslehi JJ, Amiri-Kordestani L, et al. Cardio-Oncology: Vascular and Metabolic Perspectives: A Scientific Statement From the American Heart Association. Circulation. 2019;139(13):e579-e602.

9. Curigliano G, Lenihan D, Fradley M, et al. Management of cardiac disease in cancer patients throughout oncological treatment: ESMO consensus recommendations. Ann Oncol. 2020;31(2):171-190.

10. Oliveira GH, Mukerji S, Hernandez AV, et al. Incidence, predictors, and impact on survival of left ventricular systolic dysfunction and recovery in advanced cancer patients. Am J Cardiol.2014;113(11):18931898.

11. Cardinale D, Colombo A, Bacchiani G, et al. Early detection of anthracycline cardiotoxicity and improvement with heart failure therapy. Circulation. 2015;131(22):1981-1988.

12. Ohtani K, Fujino T, Ide T, et al. Recovery from left ventricular dysfunction was associated with the early introduction of heart failure medical treatment in cancer patients with anthracycline-induced cardiotoxicity. Clin Res Cardiol. 2019;108(6):600-611.

13. Ali MT, Yucel E, Bouras S, et al. Myocardial Strain Is Associated with Adverse Clinical Cardiac Events in Patients Treated with Anthracyclines. J Am Soc Echocardiogr. 2016;29(6):522-527.e523.

14. Hatazawa K, Tanaka H, Nonaka A, et al. Baseline Global Longitudinal Strain as a Predictor of Left Ventricular Dysfunction and Hospitalization for Heart Failure of Patients With Malignant Lymphoma After Anthracycline Therapy. Circ J. 2018;82(10):2566-2574.

15. Swain SM, Whaley FS, Ewer MS. Congestive heart failure in patients treated with doxorubicin: a retrospective analysis of three trials. Cancer. 2003;97(11):2869-2879. 
16. Tanaka Y, Tanaka H, Hatazawa K, et al. Impact of hypertension on left ventricular function in patients after anthracycline chemotherapy for malignant lymphoma. Int J Cardiol. 2021;323:126-132.

17. Okada K, Mikami T, Kaga S, et al. Decreased aorto-septal angle may contribute to left ventricular diastolic dysfunction in healthy subjects. J Clin Ultrasound. 2014;42(6):341-347.

18. Nakayama T, Oshima Y, Kusumoto S, et al. Clinical features of anthracycline-induced cardiotoxicity in patients with malignant lymphoma who received a CHOP regimen with or without rituximab: A singlecenter, retrospective observational study. eJHaem . 2020;1:498-506

19. Lang RM, Badano LP, Mor-Avi V, et al. Recommendations for cardiac chamber quantification by echocardiography in adults: an update from the American Society of Echocardiography and the European Association of Cardiovascular Imaging. J Am Soc Echocardiogr.2015;28(1):1-39.e14.

20. Izumi C, Eishi K, Ashihara K, et al. JCS/JSCS/JATS/JSVS 2020 Guidelines on the Management of Valvular Heart Disease. Circ J.2020;84(11):2037-2119.

21. Pencina MJ, D'Agostino RB, Sr., D'Agostino RB, Jr., Vasan RS. Evaluating the added predictive ability of a new marker: from area under the ROC curve to reclassification and beyond. Stat Med.2008;27(2):157172; discussion 207-112.

22. Salz T, Zabor EC, de Nully Brown P, et al. Preexisting Cardiovascular Risk and Subsequent Heart Failure Among Non-Hodgkin Lymphoma Survivors. J Clin Oncol. 2017;35(34):3837-3843.

23. Wang L, Tan TC, Halpern EF, et al. Major Cardiac Events and the Value of Echocardiographic Evaluation in Patients Receiving Anthracycline-Based Chemotherapy. Am J Cardiol.2015;116(3):442-446.

24. Krasnow N. Subaortic septal bulge simulates hypertrophic cardiomyopathy by angulation of the septum with age, independent of focal hypertrophy. An echocardiographic study. J Am Soc Echocardiogr. 1997;10(5):545-555.

25. Swinne CJ, Shapiro EP, Jamart J, Fleg JL. Age-associated changes in left ventricular outflow tract geometry in normal subjects. Am J Cardiol. 1996;78(9):1070-1073.

26. Toth AB, Engel JA, McManus AM, McManus BM. Sigmoidity of the ventricular septum revisited: progression in early adulthood, predominance in men, and independence from cardiac mass. Am J Cardiovasc Pathol. 1988;2(3):211-223.

27. Aslam F, Haque A, Foody J, Shirani J. The frequency and functional impact of overlapping hypertension on hypertrophic cardiomyopathy: a single-center experience. J Clin Hypertens (Greenwich).2010;12(4):240245 .

28. Shapiro LM. Hypertrophic cardiomyopathy in the elderly. Br Heart J. 1990;63(5):265-266.

29. Pearson AC. The evolution of basal septal hypertrophy: From benign and age-related normal variant to potentially obstructive and symptomatic cardiomyopathy. Echocardiography.2017;34(7):1062-1072.

30. Chen-Tournoux A, Fifer MA, Picard MH, Hung J. Use of tissue Doppler to distinguish discrete upper ventricular septal hypertrophy from obstructive hypertrophic cardiomyopathy. Am J Cardiol.2008;101(10):14981503.

31. Belenkie I, MacDonald RP, Smith ER. Localized septal hypertrophy: part of the spectrum of hypertrophic cardiomyopathy or an incidental echocardiographic finding? Am Heart J. 1988;115(2):385-390.

32. Canepa M, Malti O, David M, et al. Prevalence, clinical correlates, and functional impact of subaortic ventricular septal bulge (from the Baltimore Longitudinal Study of Aging). Am J Cardiol.2014;114(5):796802.

33. Diaz T, Pencina MJ, Benjamin EJ, et al. Prevalence, clinical correlates, and prognosis of discrete upper septal thickening on echocardiography: the Framingham Heart Study. Echocardiography.2009;26(3):247-253. 
34. Plana JC, Galderisi M, Barac A, et al. Expert consensus for multimodality imaging evaluation of adult patients during and after cancer therapy: a report from the American Society of Echocardiography and the European Association of Cardiovascular Imaging. J Am Soc Echocardiogr. 2014;27(9):911-939.

35. Zamorano JL, Lancellotti P, Rodriguez Munoz D, et al. 2016 ESC Position Paper on cancer treatments and cardiovascular toxicity developed under the auspices of the ESC Committee for Practice Guidelines: The Task Force for cancer treatments and cardiovascular toxicity of the European Society of Cardiology (ESC). European heart journal. 2016;37(36):2768-2801.

36. Li T, Singal PK. Adriamycin-induced early changes in myocardial antioxidant enzymes and their modulation by probucol. Circulation. 2000;102(17):2105-2110.

\section{Hosted file}

Table 20th.docx available at https://authorea.com/users/437134/articles/538988-ventricularsigmoid-septum-as-a-risk-factor-for-anthracycline-induced-cancer-therapeutics-relatedcardiac-dysfunction-in-patients-with-malignant-lymphoma

Figure 1

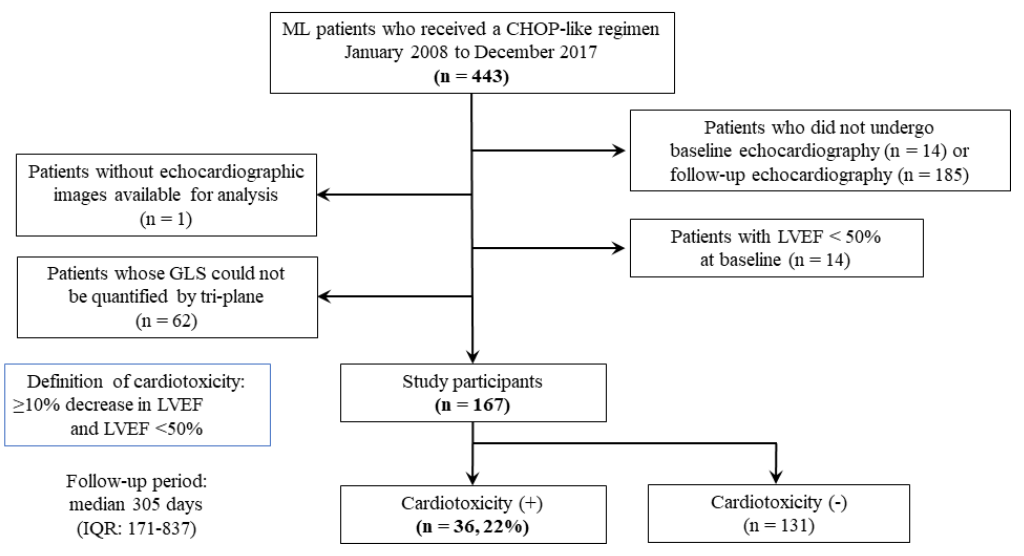

Figure 2A

Ratio of basal ventricular septal thickness compared to middle (Basal-mid ratio, B-M ratio)

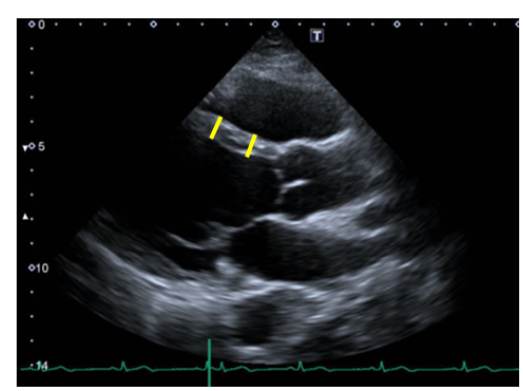

Basal-mid ratio : 1.03

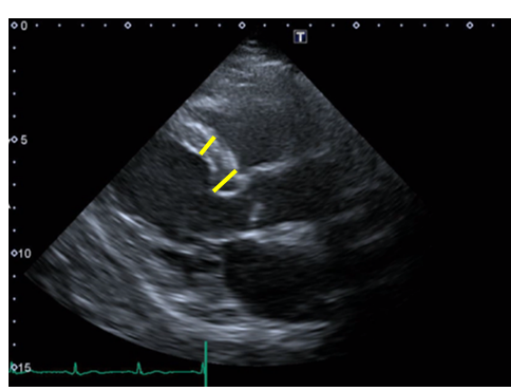

Basal-mid ratio : 1.41 
Figure 2A

Ratio of basal ventricular septal thickness compared to middle (Basal-mid ratio, B-M ratio)

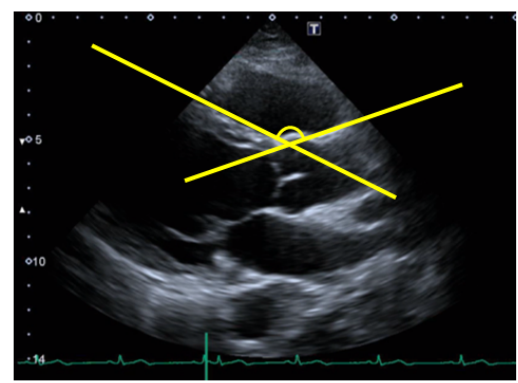

133 degree

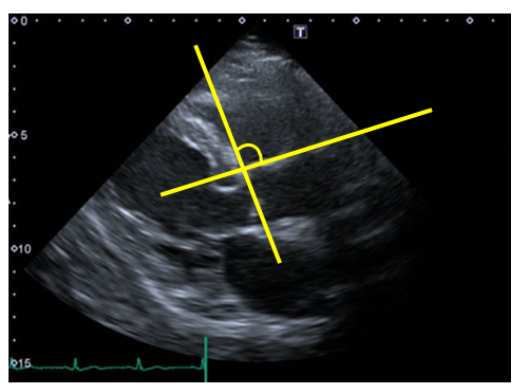

92 degree

Figure 3A

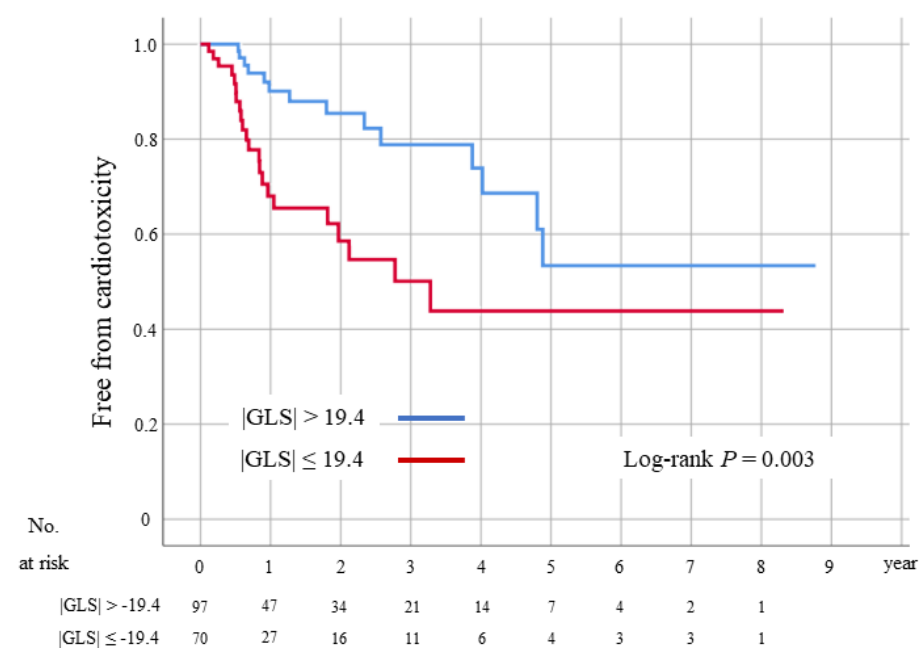


Figure 3B

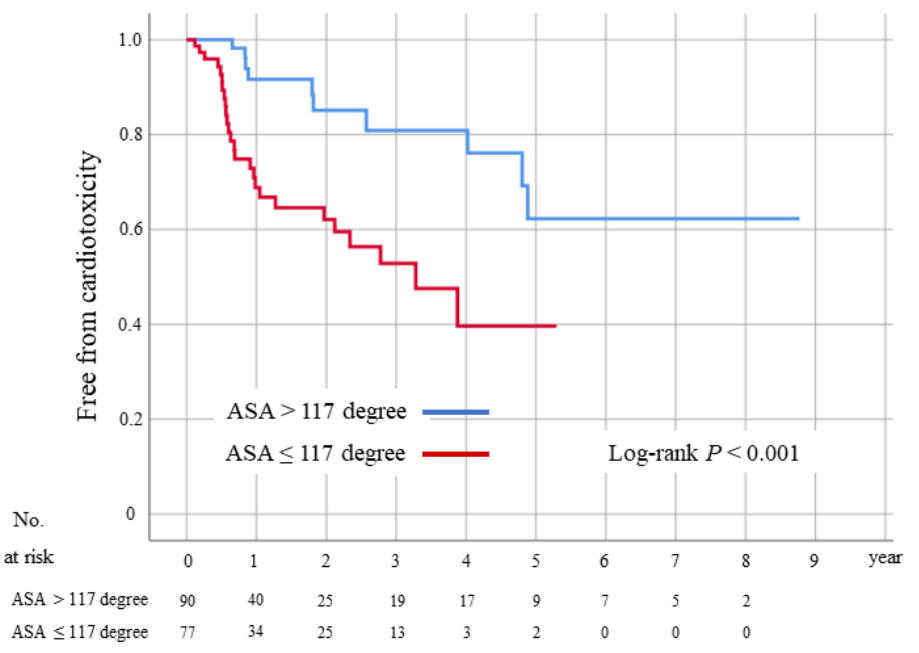

Figure 4A

C statistics of history of ischemic heart disease and GLS (model 1)

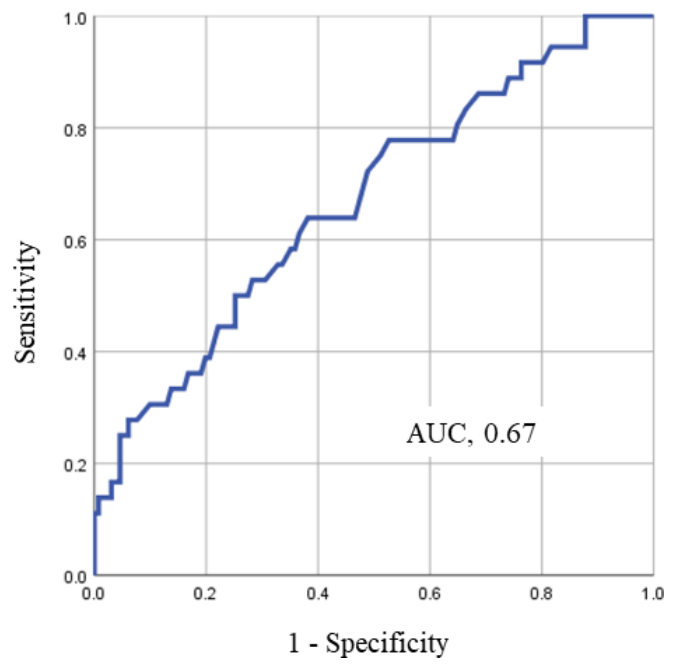


Figure 4B

C statistics of history of ischemic heart disease, GLS and ASA for Cardiotoxicity (model 2)

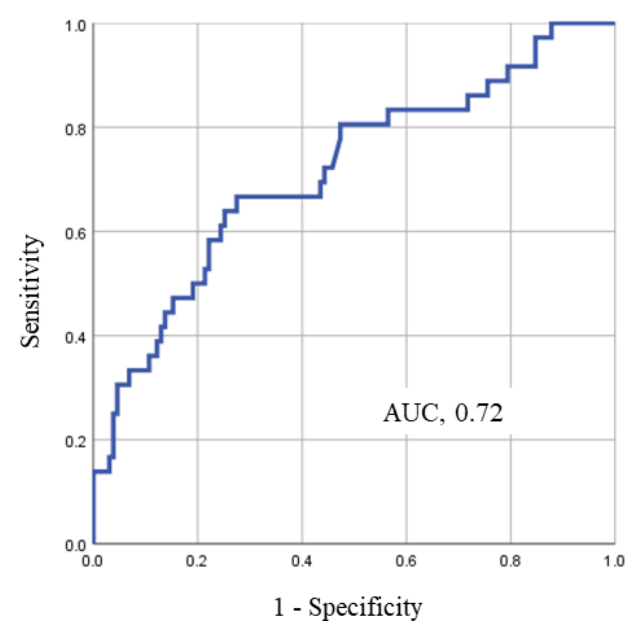

